УДК 661.721 .422

\title{
ЭКСПЕРИМЕНТАЛЬНЫЕ ИССЛЕДОВАНИЯ СИНТЕЗА МЕТАНОЛА ИЗ ЗАБАЛЛАСТИРОВАННОГО АЗОТОМ СИНТЕЗ-ГАЗА
}

\author{
Загашвили Юрий Владимирович1, \\ y.zagashvili@yandex.ru
}

\author{
Кузьмин Алексей Михайлович2,3,4, \\ kuzmin.lex@gmail.com
}

Имшенецкий Владимир Владиславович5, v.imshenetskiy@ngt-synthesis.com

\section{Лищинер Иосиф Израилевич 6 , lii48@bk.ru}

\section{Малова Ольга Васильевна 6 , mov58rus@yandex.ru}

1000 «BTP»,

Россия, 195297, г. Санкт-Петербург, Ольги Форш, 15 А, к. 1.

2 Балтийский государственный технический университет «BOEHMEX» им. Д.Ф. Устинова,

Россия, 190005, г. Санкт-Петербург, 1-я Красноармейская, 1/21.

3 ООО «Генератор синтез-газа»,

Россия, 195297, г. Санкт-Петербург, ул. Ольги Форш, 15, к. 1, кв. 49.

4 Российская Академия Естественных Наук, Россия, 190013, г. Санкт-Петербург, ул. Серпуховская, 38.

5000 «Новые газовые технологии-синтез» Sk,

Россия, 121205, г. Москва, территория инновационного центра «Сколково», ул. Нобеля, 7, этаж 2, помещение № 42.

6 Российская академия наук,

Россия, 125412, г. Москва, ул. Ижорская, 13, стр. 2.

Актуальность исследования обусловлена недостаточной изученностью процесса синтеза метанола из обогащенного азотом синтез-газа, а также отсутствием экспериментальных данных о влиянии состава синтез-газа на выход метанола-сыриа, его качество и состав отходящих газов. Проблема особенно важна для малотоннажных установок по производству метанола в промысловых условиях с использованием технологии получения синтез-газа путем парииального окисления природного газа воздухом. Цель: экспериментально подтвердить возможность синтеза метанола из обогащенного азотом синтез-газа и получить объективные оценки производительности процесса, оценить влияние температуры синтеза, состава и параметров забалластированного азотом синтез-газа на выход и качество метанола-сыриа, состав отходящих газов.

объекты: забалластированный азотом синтез-газ и его реакционная способность к синтезу метанола в зависимости от температуры синтеза, состава и параметров - мольных отношений компонентов и фракториала (модуля) - синтез-газа.

Meтоды: экспериментальные лабораторные исследования синтеза метанола с катализатором C-79-7GL компании "ZüdCheтіе» в проточном реакторе изотермического типа.

Результаты. Экспериментально подтверждена возможность синтеза метанола из обогащенного азотом синтез-газа, получаемого парциальным окислением природного газа воздухом. При давлении 4,0-4,2 МПа и объемной скорости 1100 ч$^{-1}$ для различных средних температур 210, 220 и $230{ }^{\circ} \mathrm{C}$ синтеза и двух отличающихся составом сырьевых смесей определены выходы метанола-сыриа, содержание воды и примесей в метаноле-сырце, составы и параметры отходящих из реактора газов, конверсии углерода. Уточнены оптимальная температура синтеза и допустимые вариации температуры в слое катализатора. Сформулированы рекомендации к параметрам процесса в установках с трехреакторными проточными каскадами синтеза метанола и приведена оценка их удельной производительности при использовании обогащенного азотом синтез-газа.

\section{Ключевые слова:}

Метанол, обогащенный азотом синтез-газ, газогенератор, парциальное окисление природного газа воздухом, конверсия углерода, фракториал синтез-газа.

\section{Введение}

Важной задачей современной газо- и нефтехимии является малотоннажное производство метанола, размещаемое непосредственно вблизи мест добычи углеводородного сырья [1-7]. Она особенно актуаль- на для удаленных и труднодоступных месторождений с неразвитой инфраструктурой, поскольку решает проблемы утилизации ПНГ и снижения больших логистических издержек на доставку метанола, предназначенного для ингибирования гидратообразования $[1,3-6]$. 
В России известны две малотоннажные установки производительностью 12,5 и 40,0 тыс. т метанола в год, функционирующие на Юрхаровском нефтегазоконденсатном месторождении по традиционной двухстадийной технологии, включающей на первой стадии получение синтез-газа путем паровой каталитической конверсии природного газа [8]. Для малотоннажного производства метанола целесообразно применение парциального окисления природного газа (РОХ) воздухом, достоинствами которого являются: значительное, в 3-5 раз, уменьшение массогабаритных характеристик, модульность и транспортабельность установок; применение воздуха в качестве окислителя позволяет исключить из состава оборудования дорогостоящую систему получения кислорода; снижение на 30-50 \% себестоимости синтез-газа [6-12]. И хотя генерируемый синтез-газ содержит свыше 50 об. \% азота, что существенно снижает эффективность синтеза и уменьшает производительность установок [6, 12-14], повышенная безопасность эксплуатации и возможность производства метанола непосредственно в промысловых условиях обусловливают важные дополнительные преимущества РОХ.

В продолжение работ $[14,15]$ в настоящей статье приводятся результаты новых экспериментальных исследований процесса синтеза метанола из синтез-газа с высоким содержанием азота, получаемого парциальным окислением природного газа воздухом.

\section{Описание установки}

Метанол синтезировали на лабораторной установке, схема которой приведена на рис. 1. Установка состоит из: регуляторов давления - 1, 6; расходомерарегулятора массового расхода - 2; преднагревателя - 3; реактора - 4 с загрузкой катализатора C-79-7GL компании «ZüdChemie» объемом $50 \mathrm{~cm}^{3}$ при высоте слоя 12 см; фильтра - 5; охлаждаемого сепаратора - 7; ловушки жидких продуктов - 8; счетчика газа («газовые часы») - 9. Реактор изотермического типа [14] оборудован термопарами, устанавливаемыми в аксиальный канал, для измерения температуры по слою катализатора и в пристеночный канал - для регулирования температуры в реакторе. Установка содержит баллоны с синтез-газом и азотом. Перед началом экспериментов в реактор загружают катализатор и продувают азотом из баллона. После проверки герметичности реактор нагревают до заданной температуры синтеза в рекомендуемом для свежевосстановленных медьцинкалюминиевых катализаторов диапазоне $200-230{ }^{\circ} \mathrm{C}$ $[14,16,17]$

В процессе эксперимента синтез-газ из баллона подавали под давлением 4,0-4,2 МПа через расходомеррегулятор массового расхода - 2 типа «BronkhorstElFlow» с погрешностью измерения $\pm 1 \%$ в преднагреватель - 3, где нагревали до заданной температуры. Температуру в слоях катализатора измеряли с погрешностью $\pm 0,75$ \% перемещающейся по высоте термопарой. Средняя объемная скорость синтеза составляла $1100 \mathrm{ч}^{-1}$, что эквивалентно объемному расходу $55 \cdot 10^{-3} \mathrm{HM}^{3} /$ ч. Образующиеся в процессе химических реакций продукты синтеза после фильтра - 5 поступали в сепаратор - 7, охлаждаемый циркуляционным криотермостатом. После отделения метанола-сырца в 8 отходящие газы через газовые часы - 9 поступали на анализ в хроматограф «Хромос-1000», снабженный двумя детекторами. Для анализа водорода использовали полудиффузионный детектор, газ-носитель - аргон; анализ постоянных газов проводили на проточном сдвоенном детекторе, газ-носитель - гелий. При анализе $\mathrm{CO}, \mathrm{CO}_{2}$, азота и кислорода использовали насадочную колонку «Hayesep-Q» с фазой (длиной 2 м, диаметром 3 мм, 80-100 меш.); анализ $\mathrm{H}_{2}$ проводили в изотермическом режиме при $50{ }^{\circ} \mathrm{C}$ с использованием насадочной колонки с цеолитом $\mathrm{NaX}$ (длиной 3 м, диаметром 3 мм, 60-80 меш.). Анализ жидких продуктов синтеза проводили на хроматографе «Кристаллюкс-4000М» с использованием хроматографической колонки с фазой «Db-1»; содержание воды определяли на колонке «Hayesep-Q» при $100{ }^{\circ} \mathrm{C}$.

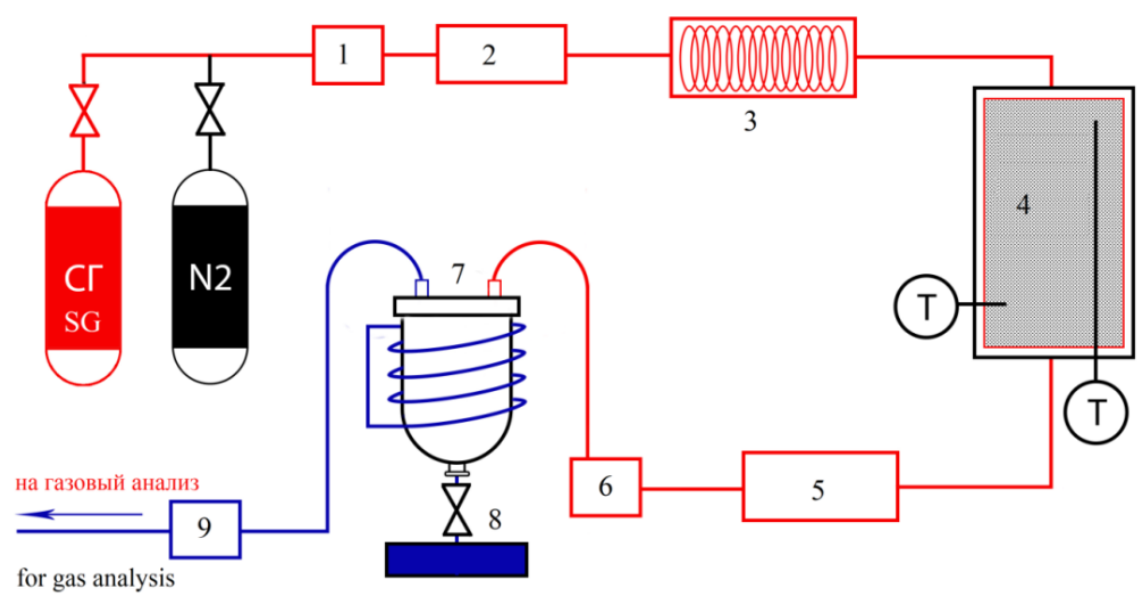

Puc. 1. Блок-схема лабораторной установки: SG - баллон с синтез-газом; N2 - баллон с азотом; 1, 6 - регулятор давления; 2 -расходомер-регулятор; 3 - преднагреватель; 4 - реактор; 5 - фильтр; 7 - сепаратор; 8 -ловушка; 9-газовые часы, $T$-термопара

Fig. 1. Block diagram of a laboratory unit: $S G$ - syngas tank; N2 - nitrogen tank; 1, 6 - pressure regulator; 2 -flow meterregulator; 3 -preheater; 4 -reactor; 5 -filter; 7 -separator; 8 -trap; 9 -gas clock, $T$-thermocouple 
Характеристики сырьевых смесей, используемых в экспериментах, приведены в табл. 1. Они совпадают с расчетными составами водородсодержащего газа на выходе комплекса получения синтез-газа, входящего в состав малотоннажной установки по производству метанола с проточным трехреакторным каскадом синтеза и выводом метанола-сырца после каждого реактора [9, 10]. Обе экспериментальных смеси соответствуют синтез-газу, полученному путем парциального окисления природного газа воздухом с коэффициентом избытка окислителя 0,38 , и отличаются значениями показателей, применяемых для оценки реакционной способности синтез-газа - мольных отношений компонентов и факториала (модуля) [16-20]. Сырьевая смесь № 1 получена без коррекции содержания газа с выхода газогенератора и имеет показатели: $\mathrm{H}_{2} / \mathrm{CO}=1,80, \mathrm{CO} / \mathrm{CO}_{2}=4,73$, модуль $\mathrm{M}=\left(\mathrm{H}_{2}-\mathrm{CO}_{2}\right) /\left(\mathrm{CO}+\mathrm{CO}_{2}\right)=1,31$; сырьевая смесь № 2 оптимизирована по критерию обеспечения требуемого значения модуля и имеет показатели: $\mathrm{H}_{2} / \mathrm{CO}=2,54, \mathrm{CO} / \mathrm{CO}_{2}=5,36, \mathrm{M}=1,99$ [15].

Таблица 1. Состав сырьевых смесей

Table 1. Mixture composition

\begin{tabular}{|c|c|c|c|c|}
\hline \multirow{2}{*}{$\begin{array}{c}\text { Компонен- } \\
\text { ты }\end{array}$} & \multicolumn{4}{|c|}{ Сырьевая смесь/Feedstock mixture } \\
\cline { 2 - 5 } Components & $\begin{array}{c}\text { № 1/no. 1 } \\
\text { сольный } \\
\text { Molar } \\
\end{array}$ & $\begin{array}{c}\text { Массовый } \\
\text { состав } \\
\text { composition }\end{array}$ & $\begin{array}{c}\text { Молs } \\
\text { composition } \\
\text { состав } \\
\text { Molar } \\
\text { composition }\end{array}$ & $\begin{array}{c}\text { Массовый } \\
\text { состав } \\
\text { Mass } \\
\text { composition }\end{array}$ \\
\hline $\mathrm{H}_{2}$ & $26,84 \pm 0,27$ & 0,025 & $30,1 \pm 0,30$ & 0,030 \\
\hline $\mathrm{CO}$ & $14,9 \pm 0,35$ & 0,194 & $11,84 \pm 0,24$ & 0,162 \\
\hline $\mathrm{CO}_{2}$ & $3,15 \pm 0,11$ & 0,065 & $2,21 \pm 0,08$ & 0,047 \\
\hline $\mathrm{N}_{2}$ & $55,11 \pm 0,95$ & 0,717 & $55,85 \pm 0,95$ & 0,762 \\
\hline $\mathrm{CH}_{4}, \mathrm{H}_{2} \mathrm{O}$ & \multicolumn{5}{|c|}{ Следы/Traces } \\
\hline
\end{tabular}

\section{Экспериментальные данные}

В табл. 2, 3 приведен материальный баланс продуктов синтеза в зависимости от средней температуры в слое катализатора и состава синтез-газа.

Таблица 2. Материальный баланс продуктов синтеза

Table 2. Material balance of synthesis products

\begin{tabular}{|c|c|c|c|c|c|c|}
\hline \multirow{4}{*}{$\begin{array}{l}\text { Наименование показателей } \\
\text { Name of indicators }\end{array}$} & \multicolumn{6}{|c|}{ Сырьевая смесь/Feedstock mixture } \\
\hline & \multicolumn{3}{|c|}{ № 1/no. 1} & \multicolumn{3}{|c|}{ № $2 /$ no. 2} \\
\hline & \multicolumn{6}{|c|}{ Температура/Temperature, ${ }^{\circ} \mathrm{C}$} \\
\hline & 200 & 210 & 230 & 200 & 210 & 230 \\
\hline $\begin{array}{l}\text { Давление, МПа } \\
\text { Pressure, MPa }\end{array}$ & 4,22 & 4,22 & 4,22 & 4,02 & 4,02 & 4,02 \\
\hline $\begin{array}{l}\text { Расход газа на входе } \\
\text { в реактор, г/ч } \\
\text { Gas flow rate at the inlet } \\
\text { of the reactor, } g / h\end{array}$ & 52,83 & 52,83 & 52,83 & 50,38 & 50,38 & 50,38 \\
\hline $\begin{array}{l}\text { Выход метанола-сырца, г/ч } \\
\text { Crude methanol yield, g/h }\end{array}$ & 0,97 & 1,61 & 0,20 & 1,15 & 1,51 & 0,40 \\
\hline $\begin{array}{l}\text { Содержание воды } \\
\text { в метаноле-сырце, \% мас. } \\
\text { Water content in raw } \\
\text { methanol, \% wt. }\end{array}$ & 22,86 & 5,37 & 3,23 & 35,23 & 5,28 & 3,87 \\
\hline $\begin{array}{l}\text { Содержание примесей } \\
\text { в метаноле-сырце, \% мас. } \\
\text { Impurity content in raw } \\
\text { methanol, \% wt. }\end{array}$ & 0,9 & 0,08 & 0,47 & 0,52 & 0,28 & 0,81 \\
\hline $\begin{array}{l}\text { Расход отходящего газа, г/ч } \\
\text { Exhaust gas flow rate, } \mathrm{g} / \mathrm{h}\end{array}$ & 51,86 & 51,22 & 52,63 & 49,23 & 48,87 & 49,98 \\
\hline
\end{tabular}

Таблица 3. Материальный баланс отходящего газа Table 3. Exhaust gas material balance

\begin{tabular}{|c|c|c|c|c|c|c|}
\hline \multirow{4}{*}{$\begin{array}{c}\text { Наименование } \\
\text { показателей } \\
\text { Name of indicators }\end{array}$} & \multicolumn{6}{|c|}{ Сырьевая смесь/Feedstock mixture } \\
\hline & \multicolumn{3}{|c|}{ № $1 /$ no. 1} & \multicolumn{3}{|c|}{ № 2/no. 2} \\
\hline & \multicolumn{6}{|c|}{ Температура/Temperature, ${ }^{\circ} \mathrm{C}$} \\
\hline & 200 & 210 & 230 & 200 & 210 & 230 \\
\hline $\begin{array}{l}\text { Расход отходящего газа, г/ч } \\
\text { Exhaust gas flow rate, } \mathrm{g} / \mathrm{h}\end{array}$ & 51,86 & 51,22 & 52,63 & 49,23 & 48,87 & 49,98 \\
\hline $\begin{array}{l}\text { Метанол, г/ч } \\
\text { Methanol, g/h }\end{array}$ & 2,97 & 3,59 & 3,84 & 2,65 & 3,60 & 3,72 \\
\hline $\begin{array}{l}\text { Содержание метанола, \% об. } \\
\text { Methanol content, \% vol. }\end{array}$ & 5,63 & 8,14 & 6,08 & 5,20 & 7,95 & 6,21 \\
\hline $\begin{array}{l}\text { Вода, г/ч } \\
\text { Water, g/h }\end{array}$ & 0,15 & 0,05 & 0,04 & 0,19 & 0,05 & 0,04 \\
\hline $\begin{array}{l}\text { Содержание вс } \\
\text { Water content, }\end{array}$ & 0,89 & 0,39 & 0,12 & 1,30 & 0,36 & 0,16 \\
\hline $\mathrm{CO}$, г/ч $(\mathrm{g} / \mathrm{h})$ & 7,47 & 5,97 & 6,78 & 5,85 & 3,94 & 4,63 \\
\hline $\begin{array}{l}\text { Содержание СО, \% об. } \\
\text { CO content, \% vol. }\end{array}$ & 12,81 & 10,84 & 11,68 & 9,94 & 7,12 & 8,01 \\
\hline $\mathrm{CO}_{2}, \Gamma / ч(\mathrm{~g} / \mathrm{h})$ & 2,58 & 3,07 & 3,29 & 1,18 & 2,07 & 2,25 \\
\hline $\begin{array}{l}\text { Содержание } \mathrm{CO}_{2}, \% \text { об. } \\
\mathrm{CO}_{2} \text { content, \% vol. }\end{array}$ & 2,82 & 3,55 & 3,61 & 1,28 & 2,38 & 2,47 \\
\hline $\mathrm{H}_{2}, \Gamma /$ ч $(\mathrm{g} / \mathrm{h})$ & 0,81 & 0,66 & 0,81 & 0,99 & 0,83 & 0,96 \\
\hline $\begin{array}{l}\text { Содержание } \mathrm{H}_{2}, \% \text { об. } \\
\mathrm{H}_{2} \text { content, \% vol. }\end{array}$ & 19,46 & 16,84 & 19,49 & 23,48 & 21,12 & 23,18 \\
\hline $\mathrm{N}_{2}, \Gamma / \mathrm{u}(\mathrm{g} / \mathrm{h})$ & 37,87 & 37,87 & 37,87 & 38,38 & 38,38 & 38,38 \\
\hline $\begin{array}{l}\text { Содержание } \mathrm{N}_{2}, \% \text { об. } \\
\mathrm{N}_{2} \text { content, \% vol. }\end{array}$ & 64,02 & 68,38 & 65,10 & 64,00 & 69,02 & 66,18 \\
\hline $\begin{array}{l}\text { Содержание примесей, г/ч } \\
\text { Impurity content, g / h }\end{array}$ & 0,01 & 0,01 & & $C_{-0}$ & T & \\
\hline
\end{tabular}

\section{Обсуждение результатов}

Анализ данных табл. 2, 3 свидетельствует об определяющем влиянии температуры катализатора на выход метанола-сырца и его качество. Максимальный абсолютный выход метанола для обеих смесей достигается при температуре $210{ }^{\circ} \mathrm{C}$ и составляет 5,12 г/ч для первой смеси и 5,03 г/ч для второй. Таким образом, удельные выходы метанола-сырца приблизительно одинаковы для обеих смесей и составляют 90 кг/ч на 1000 нм$^{3} /$ с синтез-газа или 500 кг/ч на $1000 \mathrm{Hм}^{3} /$ ч природного газа при парциальном окислении природного газа воздухом с коэффициентом избытка окислителя 0,38 .

Наблюдается высокая чувствительность выхода метанола к изменению температуры, особенно в ее нижнем диапазоне, где при уменьшении температуры синтеза с 210 до $200{ }^{\circ} \mathrm{C}$ относительный выход метанола уменьшился примерно на 30 \% для обеих смесей. Поэтому проводилась проверка изотермичности реактора синтеза путем измерения профиля температур по высоте слоя катализатора, результаты которой представлены на рис. 2. Для первой смеси при температуре нагревательного элемента $200{ }^{\circ} \mathrm{C}$ средняя температура в слое катализатора составила $195{ }^{\circ} \mathrm{C}$, при $210^{\circ} \mathrm{C}$ получили $208^{\circ} \mathrm{C}$, при $230^{\circ} \mathrm{C}$ средняя температура $227^{\circ} \mathrm{C}$; для второй смеси при тех же температурах нагревательного элемента средняя температура в слое катализатора составила соответственно: 197, 207, $227^{\circ} \mathrm{C}$. При температурах ниже $200{ }^{\circ} \mathrm{C}$ катализатор становится неэффективным [14]. Интерполируя экспериментальные данные, оптимальной средней температурой в слое катализатора C-79-7GL можно считать $215-220^{\circ} \mathrm{C}$. 
На основании данных табл. 1-3 рассчитаны параметры синтез-газа на входе и выходе реактора (табл. 4). Конверсия углерода, содержащегося в оксидах углерода исходных составов синтез-газа, в углерод метанола в экспериментах составила от 26,5 до $45,6 \%$; конверсия СО изменяется от 27 до 51,6 \%. Максимальная конверсия достигается при температуре синтеза $210{ }^{\circ} \mathrm{C}$. Учитывая коэффициенты чувстви- тельности выхода метанола к температуре, можно прогнозировать достижение $50 \%$-ой конверсии по углероду и до $60 \%$ по СО при оптимальной средней температуре в слое катализатора $215-220^{\circ} \mathrm{C}$. Для повышения конверсии СО и удельной производительности катализатора по метанолу рекомендуют увеличивать объемную скорость синтеза (уменьшать время контакта) до 10000-15000 ч ${ }^{-1}[17,18]$.
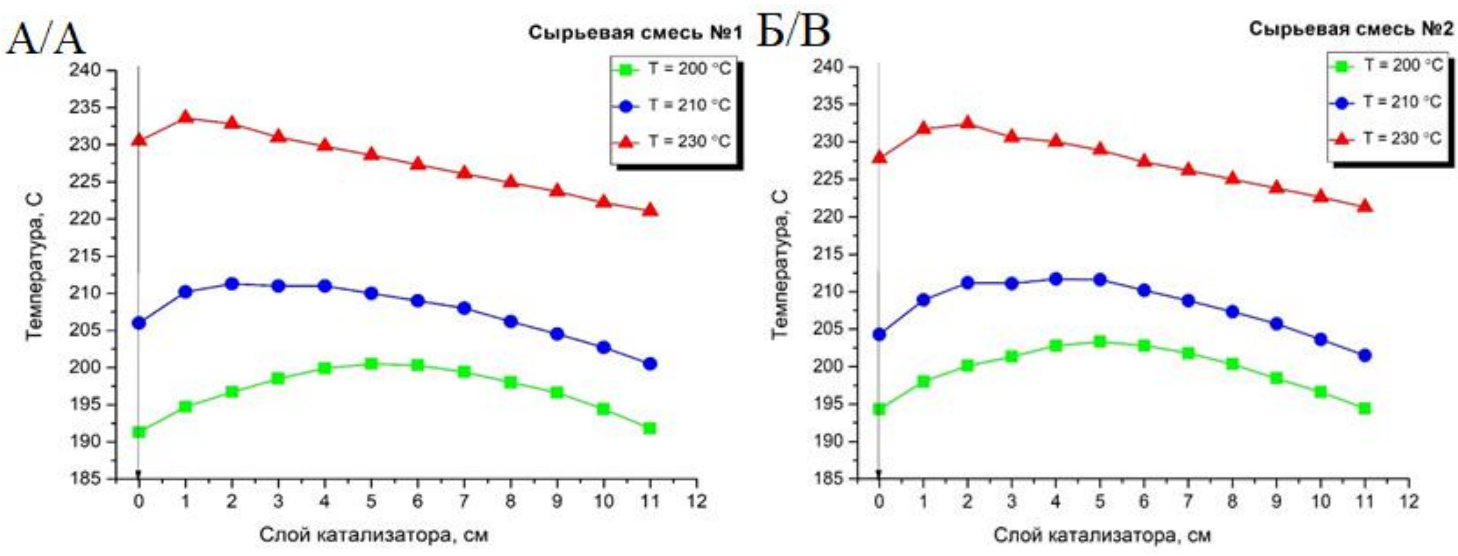

Puc. 2. Профиль температур по слою катализатора: (А) сырьевая смесь № 1, (Б) сырьевая смесь № 2

Fig. 2. Temperature profile through the bed of catalyst: (A) feedstock mixture no. 1 (B) feedstock mixture no. 2

Таблица 4. Параметры газовых потоков в реакторе

Table 4. Parameters of gas flows in the reactor

\begin{tabular}{|c|c|c|c|c|c|c|}
\hline \multirow{4}{*}{ Показатели/Indecators } & \multicolumn{6}{|c|}{ Сырьевая смесь/Feedstock mixture } \\
\hline & \multicolumn{3}{|c|}{ № $1 /$ no. 1} & \multicolumn{3}{|c|}{ № 2/no. 2} \\
\hline & \multicolumn{6}{|c|}{ Температура, ${ }^{\circ} \mathrm{C} /$ Temperature, ${ }^{\circ} \mathrm{C}$} \\
\hline & 200 & 210 & 230 & 200 & 210 & 230 \\
\hline $\begin{array}{l}\text { Расход СО на входе, г/ч } \\
\text { CO mass flow rate at the inlet, } \mathrm{g} / \mathrm{h}\end{array}$ & 10,24 & 10,24 & 10,24 & 8,14 & 8,14 & 8,14 \\
\hline $\begin{array}{l}\text { Расход С из СО на входе, г/ч } \\
\mathrm{C} \text { mass flow rate in } \mathrm{CO} \text { at the inlet, } \mathrm{g} / \mathrm{h}\end{array}$ & 4,39 & 4,39 & 4,39 & 3,49 & 3,49 & 3,49 \\
\hline $\begin{array}{l}\text { Расход } \mathrm{CO}_{2} \text { на входе, } г / \mathrm{u} \\
\mathrm{CO}_{2} \text { mass flow rate at the inlet, } \mathrm{g} / \mathrm{h}\end{array}$ & 3,40 & 3,40 & 3,40 & 2,39 & 2,39 & 2,39 \\
\hline $\begin{array}{l}\text { Расход С из } \mathrm{CO}_{2} \text { на входе, } \Gamma / 4 \\
\mathrm{C} \text { mass flow rate in } \mathrm{CO}_{2} \text { at the inlet, } \mathrm{g} / \mathrm{h}\end{array}$ & 0,93 & 0,93 & 0,93 & 0,65 & 0,65 & 0,65 \\
\hline $\begin{array}{l}\text { Расход СО на выходе, } Г / ч \\
\text { CO mass flow rate at the output, } \mathrm{g} / \mathrm{h}\end{array}$ & 7,47 & 5,97 & 6,78 & 5,85 & 3,94 & 4,63 \\
\hline $\begin{array}{l}\text { Расход С в СО на выходе, } г / \mathrm{u} \\
\mathrm{C} \text { mass flow rate in } \mathrm{CO} \text { at the output, } \mathrm{g} / \mathrm{h}\end{array}$ & 3,20 & 2,56 & 2,91 & 2,51 & 1,69 & 1,98 \\
\hline $\begin{array}{l}\text { Расход } \mathrm{CO}_{2} \text { на выходе, } Г / ч \\
\mathrm{CO}_{2} \text { mass flow rate at the output, } \mathrm{g} / \mathrm{h}\end{array}$ & 2,58 & 3,07 & 3,29 & 1,18 & 2,07 & 2,25 \\
\hline $\begin{array}{l}\text { Расход С в } \mathrm{CO}_{2} \text { на выходе, } \Gamma / ч \\
\mathrm{C} \text { mass flow rate in } \mathrm{CO}_{2} \text { at the output, } \mathrm{g} / \mathrm{h}\end{array}$ & 0,70 & 0,84 & 0,90 & 0,32 & 0,56 & 0,61 \\
\hline $\begin{array}{l}\text { Конверсия СО, \% } \\
\text { Carbon monoxide conversion, \% }\end{array}$ & 27,04 & 41,69 & 33,78 & 28,10 & 51,58 & 43,10 \\
\hline $\begin{array}{l}\text { Конверсия } \mathrm{CO}_{2}, \% \\
\text { Carbon dioxide conversion, \% }\end{array}$ & 24,15 & 9,75 & 3,28 & 50,56 & 13,26 & 5,72 \\
\hline $\begin{array}{l}\text { Конверсия C, \% } \\
\text { Carbon conversion, \% }\end{array}$ & 26,54 & 36,12 & 28,46 & 31,63 & 45,55 & 37,22 \\
\hline $\begin{array}{l}\mathrm{CO} / \mathrm{CO}_{2} \text { на входе } \\
\mathrm{CO} / \mathrm{CO}_{2} \text { at the reactor inlet }\end{array}$ & 4,73 & 4,73 & 4,73 & 5,36 & 5,36 & 5,36 \\
\hline $\begin{array}{l}\mathrm{CO} / \mathrm{CO}_{2} \text { на выходе } \\
\mathrm{CO} / \mathrm{CO}_{2} \text { output from the reactor }\end{array}$ & 4,54 & 3,06 & 3,24 & 7,77 & 2,99 & 3,24 \\
\hline $\begin{array}{l}\mathrm{H}_{2} / \mathrm{CO} \text { на входе } \\
\mathrm{H}_{2} / \mathrm{CO} \text { at the reactor inlet }\end{array}$ & 1,80 & 1,80 & 1,80 & 2,54 & 2,54 & 2,54 \\
\hline $\begin{array}{l}\mathrm{H}_{2} / \mathrm{CO} \text { на выходе } \\
\mathrm{H}_{2} / \mathrm{CO} \text { output from the reactor }\end{array}$ & 1,52 & 1,55 & 1,67 & 2,36 & 2,97 & 2,89 \\
\hline $\begin{array}{l}\text { Модуль } M \text { на входе } \\
\text { Module } M \text { at the reactor inlet }\end{array}$ & 1,31 & 1,31 & 1,31 & 1,99 & 1,99 & 1,99 \\
\hline $\begin{array}{l}\text { Модуль } M \text { на выходе } \\
\text { Module } M \text { output from the reactor }\end{array}$ & 1,06 & 0,92 & 1,04 & 1,98 & 1,97 & 1,98 \\
\hline
\end{tabular}


Исследования синтеза метанола в забалластированном азотом синтез-газе с низкими отношениями $\mathrm{H}_{2} / \mathrm{CO}$, малыми значениями модуля $M$ и достаточно высокими отношениями $\mathrm{CO} / \mathrm{CO}_{2}$ представляют большой интерес для малотоннажного производства с использованием технологии РОХ. Это обусловлено тем, что в синтез-газе, получаемом парциальным окислением природного газа воздухом, отношение $\mathrm{H}_{2} / \mathrm{CO}=1,6-1,8$ зависит главным образом от коэффициента избытка окислителя и меньше рекомендуемых значений $\mathrm{H}_{2} / \mathrm{CO}=2,2-2,6$ [15], отношения объемных концентраций оксидов углерода $\mathrm{CO} / \mathrm{CO}_{2}>4,5$ благоприятны для синтеза метанола [16-19] и практически не зависят от коэффициента избытка окислителя [15], величины модуля $M<1,5$ значительно меньше оптимальных $M=2,0-2,3$ [15-20].

Влияние состава и показателей синтез-газа в зависимости от температуры проявляется неоднозначно. Так, при температуре синтеза $210^{\circ} \mathrm{C}$ обе сырьевые смеси имеют идентичные выходы метанола, минимальные концентрации органических примесей и максимальные степени конверсии углерода. Достаточно высокие отношения $\mathrm{CO} / \mathrm{CO}_{2}>3$ сохраняются в отходящих газах во всем диапазоне температур. Однако показатели $\mathrm{H}_{2} / \mathrm{CO}$ и $M$ у отходящего газа для исходной оптимизированной смеси № 2 значительно выше и близки к оптимальным рекомендуемым значениям, что потенциально способствует более высокой степени конверсии углерода в комплексах синтеза метанола на основе многореакторных проточных каскадов с промежуточным выводом метанола-сырца после каждого реактора $[10,15,17,18]$. При средней температуре в слое катализатора $215-220{ }^{\circ} \mathrm{C}$, увеличении объемной скорости синтеза с 1100 ч $^{-1}$ до промышленной 10000 ч $^{-1}$ и рабочего давления до 5,0 МПа можно прогнозировать увеличение степени конверсии углерода в оптимизированной смеси до $60 \%$, а выхода метанола в 1,3-1,5 раза. Для увеличения выхода метанола в многореакторных проточных каскадах необходимо повышать температуру синтеза в каждом следующем по ходу потока реакторе вследствие увеличения содержания инертного азота и соответствующего уменьшения концентрации реакционных компонентов в отходящих после отделения метанола-сырца газах [10, 17].

Данные эксперимента показывают хорошее совпадение с расчетными материальными балансами установки с проточным трехреакторным каскадом синтеза с промежуточным выводом метанола-сырца после каждого реактора [15]. Например, принятые в расчетах конверсии СО соответствовали фактическим, полученным при меныших значениях рабочего давления и объемной скорости; теоретический выход метанола на $1000 \mathrm{нм}^{3} /$ ч природного газа в установке с конверсией СО в реакторах $60 \%-25 \%-10 \%$ составил

\section{СПИСОК ЛИТЕРАТУРЫ}

1. Технология переработки углеводородных газов / В.С. Арутюнов, И.А. Голубева, О.Л. Елисеев, Ф.Г. Жагфаров. - М.: Изд-во «Юрайт», 2020. - 723 с.
570 кг/ч для неоптимимизированного состава синтезгаза и 635 кг/ч для оптимизированного состава.

Качество метанола-сырца зависит от содержания в нем воды и органических примесей. С увеличением температуры синтеза содержание воды в метанолесырце резко уменышается с высоких концентраций для обеих смесей при температуре $200{ }^{\circ} \mathrm{C}$ до минимальных значений при $230{ }^{\circ} \mathrm{C}$. Эта закономерность особенно сильно проявляется в нижнем диапазоне рабочих температур. Представляет интерес динамика изменения концентрации примесей в зависимости от температуры, имеющая параболическую форму с выраженным минимумом при $210^{\circ} \mathrm{C}$.

\section{Заключение}

Экспериментально подтверждена возможность синтеза метанола из обогащенного азотом синтез-газа, получаемого парциальным окислением природного газа воздухом. Показатели качества синтеза в определяющей степени зависят от температуры катализатора. При средней температуре в слое катализатора $207^{\circ} \mathrm{C}$, объемной скорости 1100 ч $^{-1}$ и давлении 4,0-4,2 МПа на катализаторе C-79-7GL получены следующие показатели процесса: удельный выход метанола-сырца составляет 500 кг на 1000 м $^{3}$ природного газа; содержание воды менее 5,0 \% масс.; конверсия углерода, содержащегося в оксидах углерода исходного синтез-газа, в углерод метанола составила от 27 до 45,6 \%; содержание примесей в метаноле-сырце минимально. Содержание воды в метаноле-сырце сильно зависит от температуры в слое катализатора, особенно в нижнем диапазоне рабочих температур, поэтому необходимо предъявлять высокие требования к изотермичности реактора синтеза. При оптимальной средней температуре в слое катализатора $215-220{ }^{\circ} \mathrm{C}$, повышении объемной скорости до 10000 ч $^{-1}$ и рабочего давления до $5 \mathrm{MПа}$ прогнозируемое увеличение конверсии углерода и выхода метанола составит 30-50\%.

Показатели качества исходного синтез-газа $\left(\mathrm{H}_{2} / \mathrm{CO}, M, \mathrm{CO} / \mathrm{CO}_{2}\right)$ оказывают значительное влияние на характеристики газа, отходящего после отделения метанола-сырца. Мольные отношения оксидов углерода в отходящем газе зависят в основном от температуры синтеза и сохраняют достаточно высокие значения $\mathrm{CO} / \mathrm{CO}_{2}>3$, способствующие его высокой реакционной способности. Следует отметить увеличение $\mathrm{H}_{2} / \mathrm{CO}$ и сохранение оптимальных значений $M$ в отходящих газах при использовании оптимизированной сырьевой смеси № 2 в сравнении с неоптимизированным составом смеси № 1. Это свидетельствует о потенциально более высокой конверсии углерода в оптимизированном составе при синтезе метанола в многореакторных проточных каскадах и обусловливает возможность оптимизации количества реакторов.

2. Wernicke H.-J., Plass L., Schmidt F. Methanol generation // Methanol: the basic chemical and energy feedstock of the future. Berlin: Springer-Verlag Berlin Heidelberg, 2014. - P. 51-301.

3. Исследование состояния и перспектив направлений переработки нефти и газа, нефти- и газохимии в РФ / В.И. Фейгин, О.Б. Брагинский, С.А. Заболотский, И.Г. Кукушкин, А.В. Ма- 
евский, Н.И. Масленников, Ю.Г. Рыков. - М.: Изд-во «Эконинформ», 2011. - $806 \mathrm{c}$.

4. Westerterp K.R. New methanol processes // Energy efficiency in process technology / Ed. by P.A. Pilavachi. - Dordrecht: Springer, 1993. - P. 1142-1153.

5. Arutyunov V.S., Strekova L.N. Kinetic features and technological perspectives of the partial oxidation of light alkanes, Russia, Moscow // Russian Journal of Physical Chemistry. -2012. V. 6 (4). - P. 486-497.

6. Арутюнов В.С., Савченко В.И., Седов И.В. О перспективах промысловых газохимических технологий на основе азотсодержащего синтез-газа // Нефтегазохимия. - 2016. - № 4. C. 14-23.

7. Prodan V.D., Klyushenkova M.I., Borodacheva E.I. Low-tonnage methanol production, Russia // Chemical and Petroleum Engineering - 2013. - V. 49 (7-8). - P. 443-446.

8. Кемалов Р.А., Кемалов А.Ф. Технологии получения и применения метанола. - Казань: Изд-во Казан. ун-т, 2016. - 167 с.

9. Комплекс получения синтез-газа для малотоннажного производства метанола / Ю.В. Загашвили, В.Н. Ефремов, А.М. Кузьмин, И.И. Лищинер // Нефтегазохимия. - 2017. № 1. - C. 19-26.

10. Малотоннажная установка получения метанола: пат. Рос. Федерации № 176510, заявл. 20.06.2017; опубл. 22.01.2018. Бюл. № $3 .-3 \mathrm{c}$.

11. Palguandi I.J. Syngas: production, applications, and environmental impact. - New York: Nova Science Publishers, 2013. - 365 p.

12. Experimental study and kinetics modeling of partial oxidation reactions in heavily sooting laminar premixed methane flames,
China, Beijing / Q. Li, T. Wang, Y. Liu, D. Wang // Chemical Engineering Journal. - 2012. - V. 207-208. - № 1. - P. 235-244.

13. Лапидус А.Л., Елисеев О.Л., Крючков М.В. Получение углеводородов из синтез-газа, забалластированного азотом // Технология нефти и газа. - 2011. - № 5. - С. 9-12.

14. Synthesizing methanol from nitrogen-ballasted syngas, Russia, Moscow / I.I. Lishchiner, O.V. Malova, A.L. Tarasov, V.M. Maslennikov, Y.A. Vyskubenko, L.S. Tolchinskii, Y.L. Dolinskii // Catalysis in Industry. - 2010. - V. 2. - № 4. P. 368-373.

15. Загашвили Ю.В., Кузьмин А.М. Влияние состава водородсодержащего газа на выход метанола // Известия Томского политехнического университета. Инжиниринг георесурсов. 2020. - Т. 331. - № 10. - C. 187-195.

16. Технология синтетического метанола / М.М. Караваев, В.Е. Леонов, И.Г. Попов, Е.Т. Шепелев. - М.: Изд-во: «Химия», 1984. - $240 \mathrm{c}$.

17. Розовский А.Я., Лин Г.И. Теоретические основы процесса синтеза метанола. - М.: Изд-во «Химия», 1990. - 272 с.

18. Синтез метанола в системе проточных реакторов / А.В. Черепнова, А.А. Лендер, А.Г. Краснянская, Н.А. Бондарева // Катализ и нефтехимия. - 2000. - № 5-6. - С. 69-74.

19. Autothermal reforming technology for modern large-scale methanol plants / P.J. Dahl, T.S. Christensen, S. Winter-Madsen, S.M. KingProven. - Paris. Nitrogen+Syngas, 2014. - P. 1-12.

20. Appl M. Ammonia, methanol, hydrogen, carbon monoxide. Modern production Technologies. London: Nitrogen, 1997. $140 \mathrm{p}$.

Поступила 30.11.2020 2.

\section{Информация об авторах}

Загашвили Ю.В., доктор технических наук, профессор, генеральный директор ООО «ВТР».

Кузьмин A.M., кандидат технических наук, доцент кафедры двигателей и энергоустановок летательных аппаратов, Балтийский государственный технический университет «ВОЕНМЕХ» им. Д.Ф. Устинова; генеральный директор ООО «Генератор синтез-газа»; советник Российской Академии Естественных Наук.

Имшенецкий В.B., технический директор ООО «Новые газовые технологии-синтез» Sk.

Лищинер И.И., старший научный сотрудник, кандидат химических наук, Объединенный институт высоких температур Российской академии наук.

Малова O.B., кандидат химических наук, старший научный сотрудник, Объединенный институт высоких температур Российской академии наук. 
UDC 661.721 .422

\title{
EXPERIMENTAL STUDIES OF METHANOL SYNTHESIS FROM NITROGEN-BALLASTED SYNTHESIS GAS
}

\author{
Yuriy V. Zagashvili', \\ y.zagashvili@yandex.ru
}

\author{
Aleksey M. Kuzmin ${ }^{2,3}$, \\ kuzmin.lex@gmail.com \\ Vladimir V. Imshenetsky ${ }^{4}$, \\ v.imshenetskiy@ngt-synthesis.com \\ losif I. Lishchiner ${ }^{5}$, \\ mov58rus@yandex.ru \\ Olga V. Malova 5 , \\ mov58rus@yandex.ru \\ 1 LLC «HTR» \\ 15/1, Olga Forsh street, Saint-Petersburg, 195297, Russia. \\ 2 Baltic State Technical University, \\ 1/21, 1st Krasnoarmeyskaya street, Saint-Petersburg, 190005, Russia. \\ 3 LLC «GSG», \\ 15/1, Olga Forsh street, Saint-Petersburg, 195297, Russia. \\ 4 LLC «NTG-synthesis» Sk, \\ r. 42, fl. 2, 7, Nobel street, territory of the Skolkovo innovation center, Moscow, 121205, Russia. \\ 5 Joint Institute of high temperatures of the Russian Academy of Sciences, \\ 13/2, Izhorskaya street, Moscow, 125412, Russia.
}

The research relevance is caused by the lack of knowledge of methanol synthesis from nitrogen-rich synthesis gas, as well as the lack of experimental data about the effect of the synthesis gas composition on crude methanol, its quality, quantity and the composition of tale gases. The problem is particularly important for low-tonnage methanol production plants in field conditions using the technology of synthesis gas production by partial oxidation of natural gas with air.

The aim of the research is to confirm experimentally the possibility of methanol synthesis from nitrogen-rich synthesis gas and to obtain objective estimates of productivity, to evaluate the effect of the temperature of the synthesis, composition and parameters of nitrogenblasted synthesis gas on the quantity and quality of crude methanol, and the composition of tale gases.

Objects: nitrogen-rich synthesis gas and its reactivity to the synthesis of methanol depending on the synthesis temperature, composition and parameters of synthesis gas - molar ratios of components and factorial (module).

Methods: experimental laboratory studies of methanol synthesis with the «ZüdChemie» C-79-7GL catalyst in an isothermal flow reactor.

Results. The possibility of synthesizing methanol from nitrogen-rich synthesis gas obtained by partial oxidation of natural gas with air has been experimentally confirmed. At pressure of 4,0-4,2 MPa, volume velocity of $1100 \mathrm{~h}^{-1}$, for various average temperatures of 210,220 , and $230^{\circ} \mathrm{C}$ of synthesis and two different raw mixtures the authors have determined the yields of crude methanol, the content of water and impurities in crude methanol, the compositions and parameters of gases leaving the reactor, and the carbon conversion. The optimal synthesis temperature and acceptable temperature variations in the catalyst layer are specified. The paper introduces the recommendations for process parameters in the three-reactor flow units of methanol synthesis and the estimation of their productivity when using the nitrogen-rich synthesis gas.

\section{Key words:}

Methanol, nitrogen-rich synthesis gas, gas generator, partial air oxidation of natural gas, carbon conversion, factorial of synthesis gas.

\section{REFERENCES}

1. Arutyunov V.S., Golubeva I.A., Eliseev O.L., Zhagfarov F.G Tekhnologiya pererabotki uglevodorodnyh gazov [Technology of hydrocarbon gas processing]. Moscow, Yurayt Publ., 2020. 723 p.

2. Wernicke H.-J., Plass L., Schmidt F. Methanol generation. Methanol: the basic chemical and energy feedstock of the future. Berlin, Springer-Verlag Berlin Heidelberg, 2014. pp. 51-301.

3. Feigin V.I., Braginsky O.B., Zabolotsky S.A., Kukushkin I.G., Mayevsky A.V., Maslennikov N.I., Rykov Yu.G. Issledovanie sostoyaniya i perspektiv napravleniy pererabotki nefti $i$ gaza, nefti- $i$ gazokhimii $v R F$ [Research on the state and prospects of oil and gas processing, oil and gas chemistry in the Russian Federation]. Moscow, Ekon-inform Publ., 2011. 806 p.

4. Westerterp K.R. New methanol processes. Energy efficiency in process technology. Ed. by P.A. Pilavachi. Dordrecht, Springer, 1993. pp. 1142-1153.

5. Arutyunov V.S., Strekova L.N. Kinetic features and technological perspectives of the partial oxidation of light alkanes, Russia, Moscow. Russian Journal of Physical Chemistry, 2012, vol. 6, pp. 486-497.

6. Arutyunov V.S., Savchenko V.I., Sedov I.V., On the prospects of commercial gas chemical technologies based on nitrogencontaining synthesis gas. NefteGazoKhimiya, 2016, no. 4, pp. 14-23. In Rus. 
7. Prodan V.D., Klyushenkova M.I., Borodacheva E.I. Low-tonnage methanol production, Russia. Chemical and Petroleum Engineering, 2013, vol. 49 (7-8), pp. 443-446.

8. Kemalov R.A., Kemalov A.F. Tekhnologii polucheniya i primeneniya metanola [Technologies for obtaining and applying methanol]. Kazan, Kazan University Publ. house, 2016. 167 p.

9. Zagashvili Yu.V., Efremov V.N., Kuzmin A.M., Lishchiner I.I. Complex for obtaining synthesis gas for small tonnage production of methanol. Neftegazokhimiya, 2017, no. 1, pp. 19-26. In Rus.

10. Zagashvili Yu.V., Efremov V.N., Kuzmin A.M. Malotonnazhnaya ustanovka polucheniya metanola [Low-tonnage methanol production unit]. Patent RF, no. 176510, 2018.

11. Palguandi I.J. Syngas: production, applications, and environmental impact. New York, Nova Science Publ., 2013. 365 p.

12. Li Q., Wang T., Liu Y., Wang D. Experimental study and kinetics modeling of partial oxidation reactions in heavily sooting laminar premixed methane flames, China, Beijing. Chemical Engineering Journal, 2012, vol. 207-208, no. 1, pp. 235-244.

13. Lapidus A.L., Eliseev O.L., Kryuchkov M.V. Hydrocarbons production from synthesis gas, ballasted with nitrogen. Science and Technology of Hydrocarbons, 2011, no. 5, pp. 9-12.

14. Lishchiner I.I., Malova O.V., Tarasov A.L., Maslennikov V.M., Vyskubenko Y.A., Tolchinskii L.S., Dolinskii Y.L. Synthesizing methanol from nitrogen-ballasted syngas, Russia, Moscow. Catalysis in Industry, 2010, vol. 2, no. 4, pp. 368-373.

15. Zagashvili Yu.V., Kuzmin A.M. Influence of hydrogen-containing gas composition on methanol yield. Bulletin of the Tomsk Polytechnic University. Geo Assets Engineering, 2020, vol. 331, no. 10, pp. 187-195. In Rus.

16. Karavaev M.M., Leonov V.E., Popov I.G., Shepelev E.T. Tekhnologiya sinteticheskogo metanola [Synthetic methanol technology]. Moscow, Khimiya Publ., 1984. 240 p.

17. Rozovskiy A.Ya., Lin G.I. Teoreticheskie osnovy protsessa sinteza metanola [Theoretical basis of methanol synthesis]. Moscow, Khimiya Publ., 1990. 272 p.

18. Cherepnova A.V., Lender A.A., Krasnianskaya A.G., Bondareva N.A. Methanol synthesis in system of flow reactors. Catalysis and petrochemistry, 2000, no. 5-6, pp. 69-74. In Rus.

19. Dahl P.J., Christensen T.S., Winter-Madsen S., KingProven S.M. Autothermal reforming technology for modern large-scale methanol plants. Paris, Nitrogen+Syngas, 2014, pp. 1-12.

20. Appl M. Ammonia, methanol, hydrogen, carbon monoxide. Modern production technologies. London, Nitrogen Publ., 1997. 140 p.

Received: 30 November 2020.

\section{Information about the authors}

Yuriy V. Zagashvili, Dr. Sc., professor, general director of LLC «HTR».

Aleksey M. Kuzmin, Cand. Sc., associated professor, Baltic State Technical University; general director LLC «GSG»; council of the Russian Academy of Natural Sciences.

Vladimir V. Imshenetsky, technical Director of LLC «NTG-synthesis».

Iosif I. Lishchiner, Cand. Sc., senior researcher of the laboratory of Joint Institute of high temperatures of the Russian Academy of Sciences.

Olga V. Malova, Cand. Sc., senior researcher of Joint Institute of high temperatures of the Russian Academy of Sciences. 